\title{
NEW ESTIMATES OF SOME RECOMBINATION FRACTIONS IN ESCHERICHIA COLI, ALLOWING FOR THE INFLUENCE OF DIFFERENTIAL VIABILITY
}

\author{
NORMAN T. J. BAILEY \\ Department of Medicine, University of Cambridge
}

Received 28.iii.5

Extensive data on recombination in Escherichia coli, $\mathrm{K}-\mathrm{I} 2$ were given by Lederberg ( 1947, p. 5 I4). Using a somewhat unsophisticated method of analysis he derived an approximate chromosome map (p. 516). Bailey (I95I), illustrating a test for differential viability in bacterial genetics with a part of Lederberg's data, showed incidentally that the segregation of $V_{\mathbf{1}}^{r} / V_{1}^{s}$ was disturbed. It is therefore desirable to reassess the original data, making due allowance for differential viability effects.

The loci under consideration were shown by Lederberg to be arranged in the order shown in fig. $\mathbf{I}$.

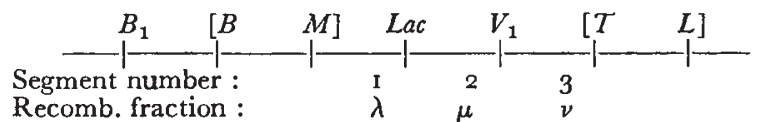

Frg. 1.

The symbols used for the various loci are as follows :-

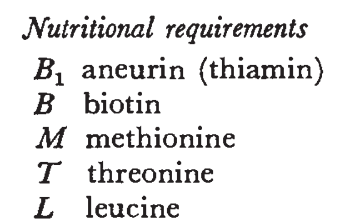

+ indicates independence

- indicates a requirement

\footnotetext{
Bacteriophage resistance

$V_{1}$ resistant to $T_{I}, T_{5}$

$r$ indicates resistance

$s$ indicates sensitivity

"Sugar" fermentation

Lac lactose
}

+ indicates ability to ferment

- indicates inability

The actual order of the loci in brackets $[B M]$ and $[T L]$ is uncertain, and these combinations are regarded as single units.

So far as the biochemical markers are concerned all the crosses under discussion were of the type

$$
B_{1}-B^{+} M^{+} T^{-} L^{-} \times B_{1}+B^{-} M^{-} T^{+} L^{+}
$$

Prototrophs were recovered either from minimal media or from plates supplemented with aneurin. In the former case recombination was "compulsory" over both the segments $B_{1}-[B M]$ and $[B M]-\lceil T L]$. In the latter case recombination was obligatory only over $[B M]-$ $[T L]$, and was "free" over $B_{1}-[B M]$. The "free" recombination 
fraction between $B_{1}$ and $[B M]$ is only of the order of 10 per cent. (Lederberg, 1947, p. 513) and it is proposed to examine both cases separately for evidence of interference.

As regards the other loci, $L a c$ and $V_{1}$, Lederberg gave data on three of the four possible mating types. All the relevant material is summarised in tables 1 and 2. The numbers (1), (2), (3) and (123)

TABLE I

Free recombination over $\mathrm{B}_{1}-[\mathrm{B} \mathrm{M}]$

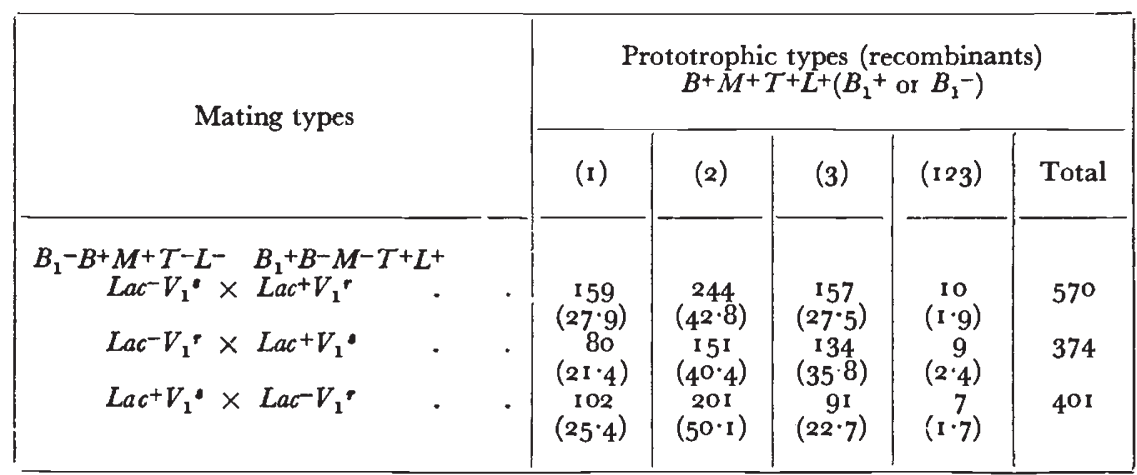

TABLE 2

Compulsary recombination over $\mathrm{B}_{1}-[\mathrm{B} \mathrm{M}]$

\begin{tabular}{|c|c|c|c|c|c|c|}
\hline \multirow{2}{*}{ Mating types } & & \multicolumn{5}{|c|}{$\begin{array}{l}\text { Prototrophic types (recombinants) } \\
\qquad B_{1}+B^{+} M^{+} T^{+} L^{+}\end{array}$} \\
\hline & & (1) & (2) & (3) & (123) & Total \\
\hline 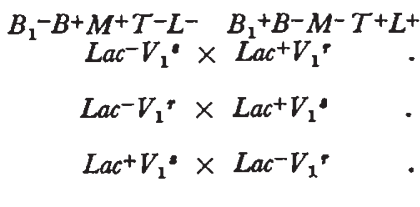 & • & $\begin{array}{c}3^{8} 7 \\
(29 \cdot 4) \\
6 \mathrm{r} \\
(19 \cdot 0) \\
28 \\
(23 \cdot 9)\end{array}$ & $\begin{array}{c}602 \\
\left(45^{\circ} \cdot 8\right) \\
145 \\
\left(45^{\circ} 0\right) \\
46 \\
\left(39^{\circ} 3\right)\end{array}$ & $\begin{array}{c}303 \\
(23 \cdot 1) \\
107 \\
(33 \cdot 2) \\
37 \\
(31 \cdot 6)\end{array}$ & $\begin{array}{c}22 \\
(1 \cdot 7) \\
9 \\
(2 \cdot 8) \\
6 \\
(5 \cdot 1)\end{array}$ & $\begin{array}{r}1314 \\
322\end{array}$ \\
\hline
\end{tabular}

refer to recombination over the first, second, third on all three of the segments shown in fig. I. The figures shown in brackets are, of course, percentages. It should be observed that the figure of 303 , appearing in column 3 for the first mating-type in table 2, was wrongly given in the original table as 203. The error was obvious on comparing the actual numbers with the percentages, and Lederberg (personal communication) tells me that 303 is correct.

The general procedure for estimating recombination fractions from this sort of data in the absence of interference was discussed by Bailey (1951). Similar methods can be used in the present instance, introducing additional parameters to represent viability effects. Let the 
recombination fractions for the three sub-segments of the segment of compulsory recombination be $\lambda, \mu$ and $\nu$ as shown in fig. I. Further, let the viability of $\mathrm{Lac}^{+}$relative to $\mathrm{Lac}^{-}$be $u$; and the viability of $V_{1} s$ relative to $V_{1}^{r}$ be $v$; the two viabilities being assumed to operate independently. Then the expectations of the four kinds of prototrophs for the first mating-type in tables $I$ and 2 are as shown in table 3 .

TABLE 3

Expectations for first mating-type

\begin{tabular}{|c|c|c|c|c|}
\hline \multirow{2}{*}{ Mating type } & \multicolumn{4}{|c|}{ Prototrophic types (recombinants) } \\
\hline & (1) $\mathrm{Lac}^{+} V_{1}{ }^{\mathrm{r}}$ & (2) $\mathrm{Lac}^{-} V_{1}{ }^{\top}$ & (3) $L a c^{-} V_{1}$ & (1 23) $L a c^{+} V_{1}$ \\
\hline $\begin{aligned} B_{1}-B^{+} M^{+} T^{-} L^{-} & ; \operatorname{Lac}^{-} V_{1}{ }^{3} \\
\times & \\
B_{1}+B^{-} M^{-} T^{+} L^{+} ; & L_{a c}+V_{1}\end{aligned}$ & $\begin{array}{c}u \lambda(1-\mu)(1-v) \\
\times n_{1} / R_{1}\end{array}$ & $\begin{array}{c}(\mathrm{I}-\lambda) \mu(\mathrm{I}-\nu) \\
\times n_{1} / R_{1}\end{array}$ & $\begin{array}{c}v(\mathrm{I}-\lambda)(\mathrm{I}-\mu) \nu \\
\times n_{1} / R_{1}\end{array}$ & $\begin{array}{l}u v \lambda \mu \nu \\
\quad \times n_{1} / R_{1}\end{array}$ \\
\hline
\end{tabular}

$n_{1}$ is the total number of prototrophs from this type of mating and $R_{1}$ is given by

$$
R_{1}=u \lambda(\mathrm{I}-\mu)(\mathrm{I}-\nu)+(\mathrm{I}-\lambda) \mu(\mathrm{I}-\nu)+v(\mathrm{I}-\lambda)(\mathrm{I}-\mu) \nu+u v \lambda \mu \nu .
$$

Similar expressions can be written down for the other mating types. This indication of the mathematical model adopted should be sufficient to show the assumptions made. It is unnecessary to reproduce here the computational details of the maximum likelihood scoring procedure which was carried out in the usual way. The results obtained were as follows.

Considering the data in table I first,we have the maximum likelihood estimates, with attached standard errors,

$$
\left.\begin{array}{rlrl}
\lambda_{1} & =\mathrm{I} 6 \cdot 28 \pm \mathrm{I} \cdot 30 \text { per cent. } & u_{1}=\mathrm{I} \cdot 038 \pm 0.067 \\
\mu_{1}=25.64 \pm \mathrm{I} \cdot 6 \mathrm{I} \quad, & v_{1}=0.775 \pm 0.05 \mathrm{I} \\
\nu_{1}=19.70 \pm \mathrm{I} \cdot 36 \quad, &
\end{array}\right\}
$$

where the subscript I indicates results with free recombination over $B_{1}-[B M]$. On the basis of the values given in (I) we can calculate the expected numbers in the various classes and then derive a goodnessof-fit $\chi^{2}$. We find $\chi_{(4)}^{2}=5 \cdot 5^{1}$, based on four degrees of freedom; this is not significant and implies a satisfactory fit. The $\mathrm{Lac}^{+} / \mathrm{Lac}^{-}$ segregation is clearly undisturbed, but there is a deficit of $V_{1}^{s}$ organisms compared with $V_{1}{ }^{r}$.

Similar calculations performed on the data of table 2 yield the results

$$
\begin{array}{rlrl}
\lambda_{2} & =\mathrm{I} 7.95 \pm \mathrm{I} \cdot 89 \text { per cent. } & u_{2}=\mathrm{I} \cdot 026 \pm 0.104 \\
\mu_{2}=27.11 \pm \mathrm{I} \cdot 80 \quad, & v_{2}=0.784 \pm 0.05 \mathrm{I} \\
\nu_{2}=19.53 \pm \mathrm{I} .55 \quad, & &
\end{array}
$$

where the subscript 2 indicates results with compulsory recombination over $B_{1}-[B M]$. This time the goodness of fit is not satisfactory with 
$\chi^{2}{ }_{(4)}=15.5$. In spite of this, however, it is remarkable that none of the estimates appearing in (2) is significantly different from the results obtained in (I). It is perhaps not unreasonable to conclude that the presence or absence of recombination over the segment $B_{1}-[B M]$ has a negligible effect on the degree of recombination observed over the segment $[B M]-[T L]:$ for the loci represented in fig. I there is, on the data available, no evidence of interference.

Owing to the poor fit of the results in (2) we are hardly justified in pooling the information in ( $\mathrm{I}$ ) and (2). The recombination fractions and corresponding map distances (derived from Haldane's formula) for the segment $[B M]-[T L]$ are shown in fig. 2 for the case of free recombination over $B_{1}-[B M]$.

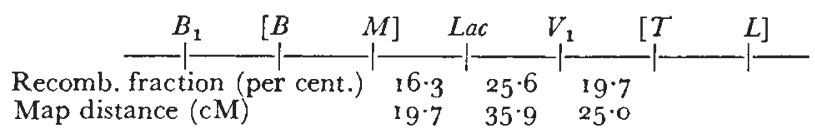

Fig. 2.-Chromosome map of Escherichia coli.

Comparing the map distances in fig. 2 with the corresponding values given by Lederberg (1947, p. $5^{16}$ ), i.e. 22, $3^{8}$ and 20 , we see that the latter values were not entirely satisfactory, the estimate for $V_{1}-[T L]$ being particularly affected by disturbance due to differential viability. Of course it should be mentioned that if there is appreciable interference- of Kosambi type, for example-then the map distances in fig. 2 will all have been seriously underestimated.

Acknowledgment.-I would like to express my thanks to Miss Eva Rowland for her assistance with the computations on which the results of this paper are based.

\section{REFERENCES}

BAILEY, NORMAN T. J. 195I. The estimation of linkage in bacteria. Heredity, 5, III-124.

LEDERBERG, JOSHUA. 1947. Gene recombination and linked segregations in Escherichia coli. Genetics, 32, 505-525. 